\title{
Quantitative Untersuchungen über den Speicherungsmechanismus von Rhodamin B, Eosin und Neutralrot in Hefezellen
}

\begin{abstract}
Von Hermann Kölbel
Aus dem Botanischen Institut der Tierärztlichen und Technischen Hochschule Hannover*

(Z. Naturforschg. 3 b, 442-453 [1948]; eingegangen am 16. Januar 1948)

Die quantitative Messung. der Farbstoffspeicherung von Modellsubstanzen und Hefezellen in Abhängigkeit von der $C_{\mathrm{H}}$. zeigen bei den drei untersuchten Farbstoffen Rhodamin B, Eosin und Neutralrot, daß die elektrische Ladung von Adsorbens und Adsorptiv eine ausschlaggebende Rolle beim Zustandekommen einer Speicherung spielt. Die Speicherungskurven der Farbstoffe Rhodamin B und Neutralrot für Hefezellen weisen eine eigenartige Feinstruktur auf, die als Ausdruck einer plasmatischen Zustandsänderung aufzufassen ist. Die früher gefundene Verschiebung des IEP der Hefezellen nach dem Abtöten konnte neuerdings bestätigt werden. Unter Zugrundelegung der untersuchten Dissoziationsverhältnisse des Neutralrots wird eine Erklärung für den Speicherungsmechanismus dieses Farbstoffes im Plasma und in der Vakuole gegeben, wobei gleichzeitig die Ursachen der Vakuolenkontraktion gedeutet werden. Auf den methodischen Wert dieser Erkenntnisse für zellphysiologische Untersuchungen wird hingewiesen. Neutralrot zeigt in seinen optischen Eigenschaften eine enge Verwandtschaft mit Acridinorange. Die kataphoretischen Untersuchungen an gefärbten und ungefärbten Hefezellen bestätigen die bereits früher gegebene Auffassung der weitgehenden Beeinflussung des elektrischen Zustandes des Adsorbens durch die Anfärbung.
\end{abstract}

$\mathrm{I}^{2}$ n Fortführung einer früheren Arbeit ${ }^{1}$ über die quantitative Speicherung des basischen Fluoreszenzfarbstoffes Acridinorange sollte mit gleicher Methodik auch die Speicherung verschiedener anderer Vitalfarbstoffe einer exakten Untersuchung unterzogen werden. Beim Acridinorange wurde als wesentlicher Faktor für das Zustandekommen einer Speicherung des Farbstoffes in der Zellmembran bzw. im Cytoplasma die in Wechselwirkung mit der Wasserstoffionenkonzentration $\left(C_{\mathrm{H}}\right)$ des Außenmediums stehende elektrische Ladung des Adsorbens erkannt. Eine Speicherung im Sinne einer Anreicherung des Farbstoffes wurde nur dann ermöglicht, wenn dem dissoziierten - also elektrisch geladenen - Farbstoff ein entgegengesetzt geladener Bindungspartner zur Verfügung stand. So ergab sich die Form der Speicherungskurve (Farbstoffspeicherung in Abhängigkeit von der $C_{\mathrm{H}}$ ) zwangsläufig aus denjenigen Kurven, die einerseits die Dissoziationsverhältnisse des Farbstoffes und andererseits den jeweiligen elektrischen Ladungszustand des Adsorbens behandeln. Die mikrokataphoretische Untersuchung zeigte, in guter Übereinstimmung mit

* Anschr. d. Verf.: Tuberkulose-Forschungsinstitut Borstel / über Bad Oldesloe.

1 H. Kölb el, Z. Naturforschg. 2 b, 382 [1947]. diesen Ergebnissen, die gänzliche oder nur teilweise Absättigung der elektrischen Ladung durch die Anfärbung.

Für die folgenden Untersuchungen wurden drei Farbstoffe ausgewählt, die jeweils einen besonderen Typ verkörpern. Das Rhodamin B ist als lipophiler Farbstoff bekannt (Strugger², Drawert ${ }^{3}$ ). Eine Abhängigkeit der Speicherung vom $p_{\mathrm{H}}$-Wert dürfte also in diesem Falle nicht zu erwarten sein, da eine solche in einer lipoiden Phase unabhängig von $\operatorname{der} C_{\mathrm{H}}$ erfolgt. Das Eosin $(\mathrm{Albach})$ ist ein ausgesprochen saurer Farbstoff. Sein Speicherungsoptimum kann also nur auf der sauren Seite des isoelektrischen Punktes (IEP) liegen. Und schließlich das Neutralrot, das als basischer Farbstoff bevorzugt für pflanzliche und tierische Objekte Verwendung findet. Die bisher aufgestellten Theorien über den Speicherungsmechanismus von Rhodamin und Neutralrot sind zahlreich und einander widersprechend, so dals eine neuerliche Bearbeitung des Problems mit möglichst exakten Methoden lohnend erschien. Dabei wurde besonderer Wert auf die Klärung

2 S. Strugger, Flora 31, 113 [1936]; Protoplasma 30, 85 [1937].

3 H. D r a w e r t, Planta 29, 376 [1939].

4 W. Albach, Protoplasma 5, 412 [1929]. 
der Dissoziationsverhältnisse der Farbstoffe gelegt, da eine genaue Kenntnis derselben unerläßliche Voraussetzung ist.

Die quantitative Messung der Farbstoffspeicherung erfolgte auf kolorimetrischem Wege mit Hilfe eines Leitz-Universalkolorimeters nach Duboscq; es wurde jeweils die Restlösung kolorimetriert. Die kolorimetrischen Eichkurven für die drei genannten Farbstoffe ergaben innerhalb des untersuchten Konzentrationsbereiches von $0,25-2,0 \cdot 10-3 \mathrm{Mol} / l$ die Gültigkeit des Beerschen Gesetzes.*

Als Versuchsobjekt dienten lebende und hitzegetötete Bäckerhefe sowie als Modellsubstanzen für Zellwand und Plasma Cellulose (Verbandwatte) bzw. Gelatine. Die von der Fabrik** bezogene frische Hefe wurde zunächst einer kurzen Reinigung unterzogen, indem sie in Leitungswasser fein suspendiert und dann 10 Min. lang zentrifugiert wurde. Die nicht zentrifugierbare Trübung, die sonst einen Fehler in der Extinktion der Meßlösung verursacht haben würde, wurde abgegossen, worauf die Hefe neuerlich suspendiert wurde. Aus dieser Suspension ( $5 \mathrm{~g}$ bei der lebenden und $2 \mathrm{~g}$ bei der toten jeweils in $50 \mathrm{~cm}^{3}$ ) wurden $5 \mathrm{~cm}^{3}$ abpipettiert und $\mathrm{zu} 45 \mathrm{~cm}^{3}$ einer gepufferten (Phosphatpuffer) Farbstofflösung zugefügt, welche dann eine Konzentration von $c=0,5 \cdot 10^{-3} \mathrm{Mol} / l$ besaß. Beim Neutralrot war nur eine solche von $c=0,25 \cdot 10^{-3} \mathrm{Mol} / l \mathrm{zu}$ verwenden, da konzentriertere Lösungen eine zu hohe Extinktion aufweisen, welche im Koiorimeter eine sehr kleine Schichtdicke erfordern würde, die unter Berücksichtigung der Meßfehlergrenze (optimal $\sim 1 \%$ ) nicht mit genügender Genauigkeit einzuhalten ist. Die Färbedauer betrug bei allen Objekten 10 Min. Die tote Hefe wurde erhalten, indem die Suspension auf dem siedenden Wasserbad erhitzt wurde, bis sie eine Temperatur von $90^{\circ} \mathrm{C}$ aufwies.

\section{I. $\mathrm{Rhodamin} B$}

Einige quantitative Vorversuche mit Modellsubstanzen zeigten eine eindeutige Abhängigkeit der Speicherung von der $C_{\mathrm{H}^{*}}$, vornehmlich im sauren Gebiet, und so lag es nahe, eine auf Grund der Konstitution (s. Abb.1) auch zu erwartende Dissoziation des Rhodamins nachzuweisen. Auf kolorimetrischem Wege gelang es allerdings nicht, eine meßbare Intensitätsverschiebung der Farbe bei wechselnden $p_{\mathrm{H}}$-Werten festzustellen. D rawert ${ }^{3}$ untersuchte verschiedene Farbstoffe - so

* Hrn. Dr. H. Friedrich-Freks a verdanke ich den Hinweis auf eine Arbeit von G. K o rtü m (Z. physik. Chem. 33, 1 [1936]), wonach das EosinAnion bei unterschiedlichen Wellenlängen verschieden starke Abweichungen vom Beerschen Gesetz zeigt. So ist z. B. schon bei $c=5 \cdot 10^{-3} \mathrm{Mol} / l$ und $\lambda=546 \mathrm{~m} \mu$ (langwellige Absorptionsbande) die Abweichung um etwa $48 \%$ größer als bei $c=3 \cdot 10^{-5}$, dagegen tritt bei $\lambda=436 \mathrm{~m} \mu$ und der gleichen Konzentration von $c=5 \cdot 10^{-3}$ die Abweichung praktisch auch Rhodamin B - kataphoretisch mit Hilfe von Filtrierpapierstreifen, die als Elektroden in die Lösung gehängt wurden. Aus der mehr oder weniger großen Anstieghöhe wurde von ihm auf den Dissoziationsgrad geschlossen. Es erscheint aber nicht berechtigt, die auf diese Weise erhaltenen Ergebnisse irgendwie quantitativ auszuwerten. Die Methode mag lediglich zur groben kataphoretischen Charakterisierung von Farbstoffen mit einfachen Dissoziationsverhältnissen dienen, da durch die unterschiedliche $C_{\mathrm{H}}$. der Farblösung auch der physikalische Zustand (elektrische Ladung, Quellungsgrad usw.) der Cellulose geändert wird, was sich ohne Zweifel auf die Steighöhe des Farbstoffes in den Papier-Elektroden auswirkt. Außerdem wird bei dieser Versuchsanordnung die Anderung des Aciditätsgrades an Elektroden außer acht gelassen, wodurch bei längerer Versuchsdauer (15 Min.!) ein zusätzlicher Fehler verursacht wird.

Da eine kataphoretische Untersuchung des Rhodamins aber notwendig war, wurde eine Glasapparatur hergestellt, die im wesentlichen aus einem U-Rohr mit zwei Hähnen und einem Schliffaufsatz zur Aufnahme der Elektroden bestand, ähnlich wie sie bereits von $\mathrm{Mich}$ a elis ${ }^{5}$ u. a. beschrieben worden ist. In Ermangelung von Edelmetallen wurde als Anode Spektralkohle verwendet, deren oberes Ende mit Paraffin umhüllt wurde. Als Kathode diente ein ebenso geschützter Eisendraht. Die beiden Elektroden tauchten in zwei Gefäße, welche zur ersten Aufnahme der sekundären Elektrolyse-Produkte entsprechend geräumig gehalten waren. Zwischen diesen beiden Gefäßen bestand außerdem eine durch einen Hahn zu öffnende Verbindung, die als Niveau-Ausgleich diente.

Zur kataphoretischen Untersuchung wurde die gepufferte wäßrige Farbstofflösung in den Scheitelteil des U-Rohres eingebracht, worauf die beiden Glashähne geschlossen wurden. Nach dem Ausspülen der Schenkel des U-Rohres wurde das Oberteil aufgesetzt. und das ganze System bei geöffnetem Niveauhahn mit der farblosen Pufferlösung gefüllt. Dann wurden die beiden Elektroden eingesetzt und beide Glashähne gleichzeitig und möglichst erschütterungsfrei geöffnet, so daß die beiden Flüssigkeiten mit scharfer Trennlinie überschichtet wurden. Nach Einschalten des Stromes wurde der Niveauhahn geschlossen und

noch nicht in Erscheinung. Die Messungen am Eosin wurden von mir mit einem Filter vorgenommen, dessen Schwerpunkt bei $\lambda=450 \mathrm{~m} \mu$ lag, so daß die Meßergebnisse in Übereinstimmung mit denjenigen von Kortüm stehen.

** Der Fa. Asmussen, Niederlassung Hannover, W. Bröckerbaum, sei auch an dieser Stelle für die freundliche Überlassung der Hefe gedankt.

${ }^{5} \mathrm{~L}$. M i chaelis, Die Wasserstoffionenkonzentration. Springer, Berlin 1926. 
die Stromstärke auf $3,0 \mathrm{~mA}$ einreguliert. Die Versuchsdauer betrug 20-30 Min.

Die so angestellten Untersuchungen ergaben unterhalb $p_{\mathrm{H}} 3,2$ eine eindeutige Wanderung des Farbstoffes in den kathodischen Schenkel des URohres, während diese oberhalb $p_{\mathrm{H}} 3,8$ in eine anodische überging; doch vollzog sich letztere etwas träger. Zwischen diesen beiden Grenzwerten verhielt sich der Farbstoff im KataphoreseVersuch indifferent. In annähernder Utbereinstimmung hiermit fand $\mathrm{Yamaha}{ }^{6}$, daß der Farbstoff im Gebiet von $p_{\mathrm{H}} 2,0-3,5$ seine kataphoretische Wanderungsrichtung umkehrt. Drawert ${ }^{3}$ hingegen konnte mit der bereits oben kritisch beleuchteten Methode keine Ånderung des kataphoretischen Verhaltens im untersuchten Gebiet von $p_{\mathrm{H}} 2-11,5$ feststellen. Er nimmt deshalb an, daß der Farbstoff nicht dissoziiert ist. Die im Molekül vorhandene Carboxylgruppe läßt aber ohne Zweifel ,eine Anderung des Dissoziationszustandes erwarten, die nicht gleichzeitig mit einer
Anderung der Farbe verbunden sein muß. Es ist anzunehmen, daß das $p_{k}$ der Carboxylgruppe bei $\sim 3,8$ liegt. Unterhalb $p_{\mathrm{H}} 3,2$ existiert der Farbstoff als Kation. Das elektrisch neutrale Verhalten zwischen den angegebenen Grenzwerten kommt aber nicht dadurch zustande, daß der-Farbstoff nicht dissoziiert ist, sondern muß auf eine intramolekulare Neutralisation zurückgeführt werden. Ein zweifach positiv geladenes Rhodamin B-Ion, das durch Protonenanlagerung entsteht, dürfte erst im stark sauren Gebiet existieren, da kolorimetrisch keine Aufhellung der Farbe bis $p_{\mathrm{H}} 2$ festgestellt werden konnte. Diese Anderung in der Farbintensität müßte sich aber durch die Protonenanlagerung ergeben, da durch die Festlegung des einsamen Elektronenpaares am Stickstoff seine Beteiligung an der Mesomerie verhindert wird. Die oberhalb $p_{\mathrm{H}} 3,8$ anodisch werdende Wanderung des Farbstoffes steht nun im Widerspruch zu den theoretisch abzuleitenden Verhältnissen:

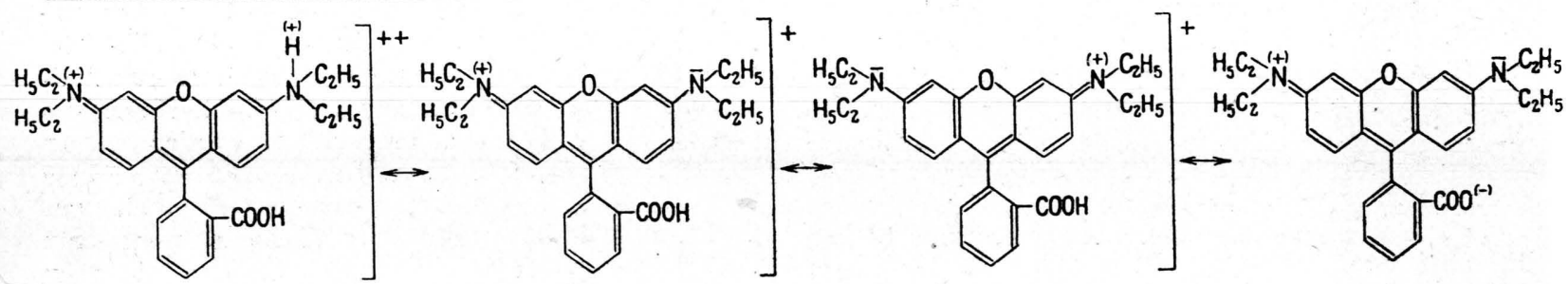

Hiernach muß das Rhodamin B auch im alkalischen Gebiet als Zwitterion vorliegen und wäre somit als Molekül elektrisch neutral. Die Erklärung dafür, daß der Kataphorese-Versuch ein anderes Resultat ergab, mag in gewissen Verunreinigungen mitRhodaminen (verwendet wurde das Rhodamin B von Merck) anderer Konstitution

( $\mathrm{G}$ oder $6 \mathrm{G}$ ) ihre Ursache haben, bei welchen eine negative Aufladung durch die Dissoziation der Carboxylgruppe und Abgabe des Protons vom Stickstoff denkbar wäre. Jedenfalls stehen die experimentellen Befunde der Kataphorese wie auch der nachfolgend wiedergegebenen Ergebnisse der Speicherungsmessungen im besseren Einklang mit folgenden Formulierungen (Rhodamin G) :

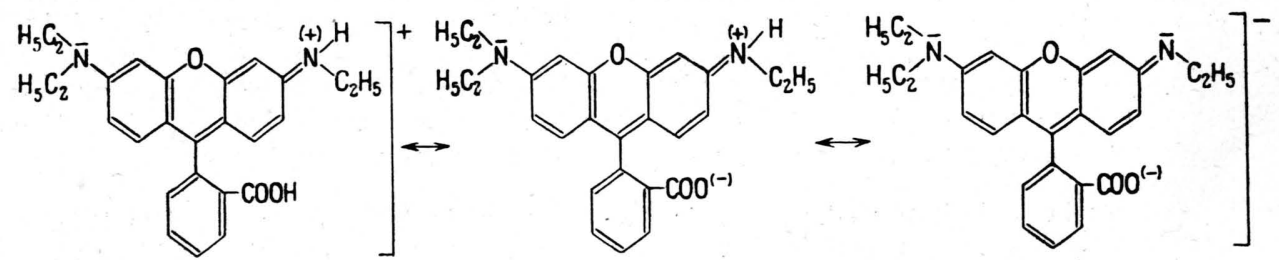

Eine endgültige Entscheidung ist aber nur durch Untersuchungen mit wirklich reinen, definierten Farbstoffen zu treffen.

Übereinstimmend mit diesen Vorstellungen las6. G. Y a m a h a, Cytologia 9, 193 [1938]. sen sich nun die qualitativen Angaben von Drawert ${ }^{3}$ und Strugger ${ }^{2}$ über die Speicherung des Rhodamins B verstehen. In einem von Strugger angegebenen Versuchsprotokoll färbt sich beispielsweise die Membran lebender Zwiebelepider- 
miszellen bei $p_{\mathrm{H}} 2,04$, während gleichzeitig das Plasma nur eine schwache Anfärbung zeigt. Bei $p_{\mathrm{H}} 4,55$ hingegen ist die Membran ungefärbt, es speichert aber jetzt das Plasma. Auch hier ergibt sich also die Anderung der Aufladung des Farbstoffes im dargelegten Sinne.

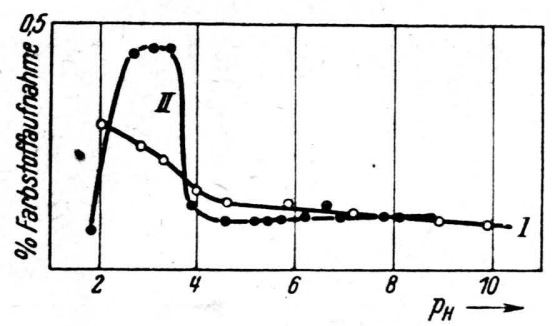

Abb. 1. Rhodamin B. Farbstoffspeicherung in Cellulose (I) und Gelatine (II).

Um nunmehr den quantitativen Verlauf der Speicherung in Abhängigkeit von der $C_{\mathrm{H}}$ festzulegen, wurde zunächst die Farbstoffaufnahme von Cellulose und Gelatine gemessen. Abb. 1 zeigt das Ergebnis.

Die Analyse dieser beiden Kurven ergibt folgendes: Die Speicherung der Cellulose (I) zeigt im Gebiet zwischen $p_{\mathrm{H}} 2$ und 4 eine deutliche Erhöhung. Unter Zugrundelegung des auf Grund der Kataphorese anzunehmenden $p_{k}$-Wertes der Carboxylgruppe von 3,8 läßt sich diese Erhöhung ohne weiteres verstehen: die positive Aufladung des Farbstoffes auf der sauren Seite dieses Gebietes bedingt seine hohe Speicherung in der Cellulose. Mit Zunahme der Dissoziation der Carboxylgruppe sinkt auch die Farbstoffbindung. Entsprechendes gilt nun für die Gelatine (II), deren IEP durch früher angestellte quantitative Speicherungsmessungen mit Acridinorange bei $p_{\mathrm{H}} 3,3$ gefunden wurde*. Theoretisch ist die Lage des Speicherungsmaximums dort zu erwarten, wo beim Vorhandensein ausreichender Mengen von negativ geladenen Farbstoffionen das Adsorbens eine möglichst hohe positive Aufladung besitzt. Die Lage dieses Punktes wäre auf $\operatorname{der} \cdot p_{\mathrm{H}}-$ Skala in unmittelbarer Nähe des Umladungsgebietes des Farbstoffes vorauszusagen, was auch tatsächlich, wie Abb. 1 zeigt, der Fall ist. Diese Messungen, lassen also auch bei diesem Farbstoff die elek-

* Die Herkunft der von uns für diese Modelluntersuchungen verwendeten Gelatine war unbekannt; gereinigte Gelatine stand nicht zur Verfugung. Die Abweichung in der Lage ihres IEP von den sonst genannten Werten $\left(\mathrm{um} p_{\mathrm{H}} 4,5\right)$ mag hierin ihre Ursache haben. trische Ladung als wesentlichen Faktor beim Speicherungsvorgang im sauren Gebiet erkennen.

Die Speicherungskurven für lebende (I) und tote (II) Hefe sind in Abb. 2 wiedergegeben. Zur Deutung der Form dieser Kurven ist die Kenntnis des IEP der Hefezellen notwendig, welcher für die lebenden bei $p_{\mathrm{H}} 6,2$ und für die toten bei $p_{\mathrm{H}} 4,8$ gefunden wurde (Kölbel ${ }^{1}$ ). Die Vitalfärbung der Hefe zeigt keine ausgeprägte Abhängigkeit von der $C_{\mathrm{H}^{*}}$. Bei der toten besteht ein Maximum bei $p_{\mathrm{H}} 3,4$, was nach oben Gesagtem verständlich wird. Die gegenseitige elektrostatische Bindung ist hier also ausschlaggebend für die Speicherung und läßt die lipophile Natur des Farbstoffes in den Hintergrund treten.

Eine auffallende Besonderheit ergibt sich im Verlauf der Speicherungskurve für tote Hefezellen bei $p_{\mathrm{H}} 6,2$ (Abb. 2). Dieser Punkt ist durch eine besonders markante Erhöhung der Speicherungsfähigkeit ausgezeichnet, für die eine Deutung bisher aussteht. Sie kann jedenfalls als spezifischer Ausdruck einer plasmatischen $\mathrm{Zu}$ standsänderung der Zellen aufgefaßt werden;

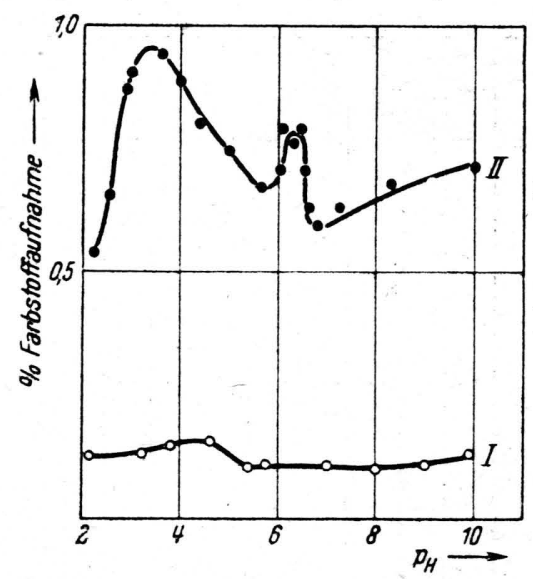

Abb. 2. Rhodamin B. Farbstoffspeicherung in lebenden (I) und toten (II) Hefezeilen.

hier kann nur umfassendes Versuchsmaterial, das in gleicher Weise mit anderen Farbstoffen gew onnen werden muß, die Möglichkeit einer Erklärung ergeben, welche dann allerdings interessante Ergebnisse erwarten läßt. Die Reproduzierbarkeit der Feinstruktur wurde besonders eingehend geprüft; es ergab sich in jedem Falle das scharf ausgeprägte Maximum bei $p_{\mathrm{H}} 6,2$.

Cellulose, Gelatine und lebende Hefezellen speichern den Farbstoff oberhalb $p_{\mathrm{H}} \sim 4$ etwa in 
gleicher Höhe, obwohl die beiden Modellsubstanzen kein lipoides System darstellen, im Gegensatz zur Hefe. Daß die toten Hefezellen imstande sind, ein Vielfaches der Farbstoffmenge der lebenden aufzunehmen, mag seinen Grund in der Änderung der Permeabilitätsbedingungen für den Farbstoff haben, doch wäre auch denkbar, daß die erhöhte Aggregation der lipoiden Substanzen - hervor- gerufen durch die Hitzekoagulation des Plasmas günstigere Lösungsbedingungen für den Farbstoff schafft.

\section{Eosin}

Als Vertreter eines sauren Farbstoffes wurde das Eosin in den Rahmen der Untersuchungen einbezogen. Die Dissoziationsverhältnisse werden durch folgende Ionenformeln klargelegt:<smiles>O=C(O)c1ccccc1-c1c2cc(Br)c(=O)c(Br)c-2oc2c(Br)c(Br)c(Br)cc12</smiles><smiles>O=C(O)c1ccccc1-c1c2cc(Br)c(=O)c(Br)c-2oc2c(Br)c(Br)c(Br)c(Br)c12</smiles>

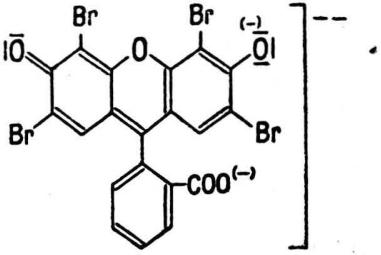

Die in Abb. 3 wiedergegebene Dissoziationskurve, die kolorimetrisch gefunden wurde, bezieht sich auf die Vorgänge an den Hydroxylgruppen, während die Lösung des Protons von der Carboxylgruppe ebenso wie beim Rhodamin natürlich keine Anderung der Farbe bedingt und somit kolorimetrisch nicht $\mathrm{zu}$ fassen ist. Gemäß der Zahl der zur Verfügung stehenden Eosin-Anionen

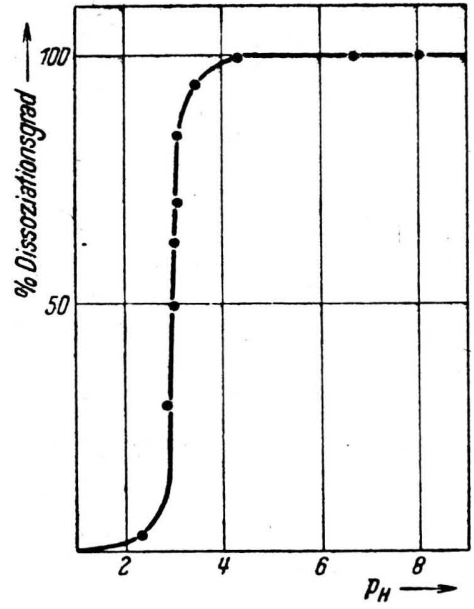

Abb. 3. Eosin. Dissoziationskurve (kolorimetrisch).

setzt die Speicherung oberhalb $p_{\mathrm{H}} 2-3$ mit hohen Werten ein (hohe positive Aufladung des Plasmas!), sie fällt dann aber schnell wieder ab, da totes wie lebendes Plasma sich mit höheren $p_{\mathrm{H}^{-}}$ Werten ihren Umladungspunkten nähern (Abb.4). Mit Hilfe dieser Kurven lassen sich allerdings keine Aussagen über die Lage des IEP machen. Wegen des eng begrenzten Speicherungsgebietes eignet sich mithin dieser Farbstoff nicht für derartige Bestimmungen. Bemerkenswert ist jedoch die verhältnismäßig hohe Speicherung des Eosins trotz der Größe (,Sperrigkeit") seines Moleküls. Wir müssen also - ähnlich wie beim Acridinorange -

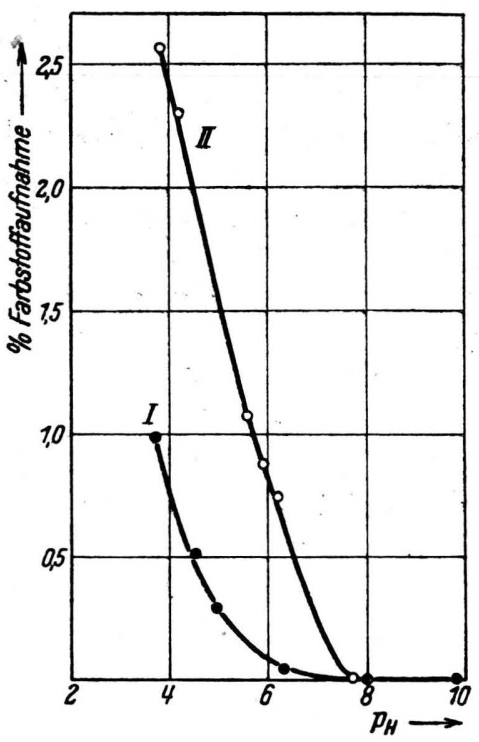

Abb. 4. Eosin. Farbstoffspeicherung in lebenden (I) und toten (II) Hefezellen.

auf eine morphologische Verwandtschaft zum Plasma schließen (vgl. auch die große Einlagerungsfähigkeit des Kalium-Fluoresceins, wovon quantitative Messungen allerdings noch nicht vorliegen; s. Döring ${ }^{7}$ und Strugger ${ }^{8}$ ).

Die basischen Farbstoffe haben nur deswegen

‘ H. Döring, Ber. dtsch. bot. Ges. 53, 415 [1935].

8 S. Strugger, Flora 32, 253 [1938]. 
in so überwiegendem Maße Verwendung in der zunächst rein empirischen biologischen Färbemethodik gefunden, weil die Speicherung von Farbstoffionen auf elektrostatischen Adsorptionsvorgängen beruht, und man gewohntermaßen im neutralen bis schwach sauren Milieu färbt, in einem $p_{\mathrm{H}}$-Bereich also, in welchem das Plasma eine negative Ladung trägt, da der IEP eines Protoplasten im allgemeinen im sauren Gebiet liegt. Die sauren Farbstoffe haben also lediglich aus diesem Grunde geringere Bedeutung erlangt und nicht etwa, weil sie sich gegenüber den basischen durch eine größere Giftwirkung auszeichnen, oder eine weniger hohe spezifische Adsorptionsfähigkeit besitzen.

\section{Neutralrot}

Die kolorimetrische. Untersuchung über den Verlauf der Farbänderung des Neutralrots in Abhängigkeit von der $C_{\mathrm{H}}$. wurde in der Absicht unternommen, die Dissoziationskurve des Farbstoffes festzulegen. Es wurde dabei - wio schon entsprechend bei den anderen Farbstoffen - die Extinktion einer Neutralrotlösung von extrem niedrigem $p_{\mathrm{H}}(1,0)$ mit derjenigen anderer Lösungen gleicher Konzentration, aber von abgestuftem $p_{\mathrm{H}}$-Wert, verglichen. Zur Erhöhung der Meßgenauigkeit wird dabei gewöhnlich ein Filter vorgeschaltet, dessen Schwerpunkt sich nach dem Absorptionsmaximum der Farblösung richtet. In unserem Falle wurde ein Grünfilter $(\lambda=5510 \AA)$ verwendet. Die so gewonnene Kurve zeigt Abb.5 (I). Das Abweichen von dem normalen (S-förmigen) Verlauf einer Dissoziationskurve erklärt sich folgendermaßen: Das Neutralrot wechselt seine Farbe mit abnehmender $C_{\mathrm{H}^{*}}$ von Blau über Purpur nach Rot, Orange und schließlich nach Gelb. Demgemäß ergibt sich der Verlauf der Kurve aus den Durchlässigkeiten des Grünfilters für den jeweiligen Farbton. Für Blau ist die $\mathrm{Ab}$ sorption geringer als für Violett (Komplementärfarbe!), folglich steigt die Kurve an. Sie fällt dann wieder infolge Utberwiegens der roten Fär- bung, die allmählich über eine gelbrote in die rein gelbe umschlägt, die eine geringe Absorption für Grün besitzt. Aus diesen Untersuchungen ergeben sich nun die wahren Dissoziationsverhältnisse.

Das „saure Salz" des Neutralrots ist blau gefärbt und das neutrale rosenrot ${ }^{9}$. Die gelbe Färbung wird allgemein als der Base zugehörig beschrieben. Verfolgt man den Dissoziationsvorgang vom Gebiet hoher $C_{\mathrm{OH}^{-}}$in dasjenige hoher $C_{\mathrm{H}^{*}}$, so wird zunächst ein Proton aufgenommen,

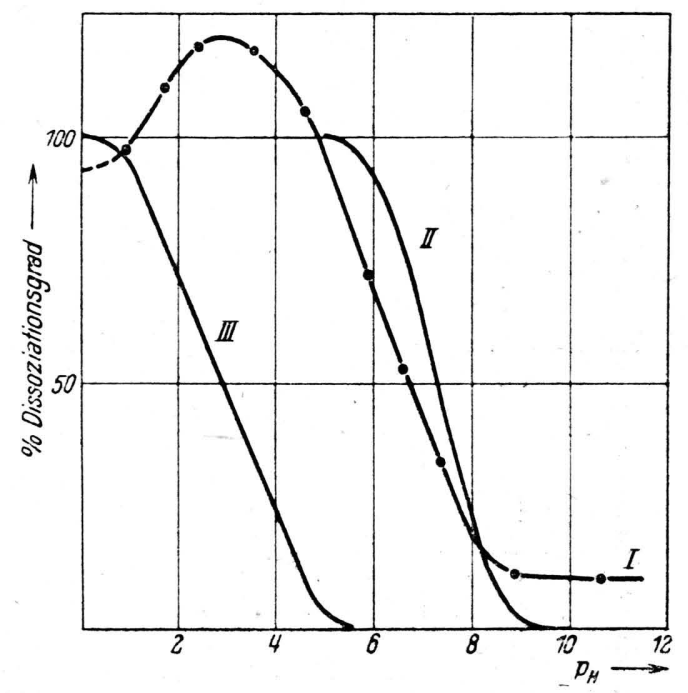

Abb. 5. Neutralrot. Relative (I) und wirkliche (II u. III) Dissoziationsverhältnisse.

wodurch ein einfach positiv geladenes - rot gefärbtes - Farbstoffion entsteht. Wenn dieser Vorgang bis zur einfachen Aufladung sämtlicher Moleküle abgeschlossen ist, wird bei weiterer Erhöhung $\operatorname{der} C_{\mathrm{H}}$ ein zweites H-Ion gebunden. Hierdurch entsteht das blau gefärbte Kation. Die kolorimetrisch gefundene Kurve löst sich also in zwei Dissoziationskurven auf. Die rein blaue Färbung deutet auf maximale Aufladung hin, während eine rein rote die Vorstufe hiervon anzeigt. Infolge des aufgezeigten Zusammenhangs muß der $p_{\mathrm{H}^{-}}$-Wert, bei welchem die unmittelbar aus der Base hervorgehende Form allein vorliegt,

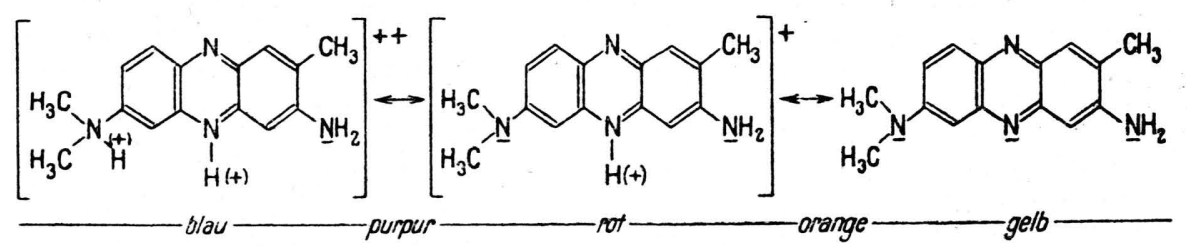

9 Beilsteins Hdb. organ. Chem., Bd. XXV, S. 401. 
den Beginn der weiteren Aufladung zur blauen bedeuten. Nimmt man in erster Näherung an, daß die Größe der Absorption des verwendeten Grünfilters für Rot und Blau etwa gleich ist, so muß dieser Wert um $p_{\mathrm{H}} 5,3$ liegen. Unter der weiteren Annahme, daß das Maximum der Kurve I durch die Absorption gleicher Anteile von einfach und doppelt geladenen Farbstoffkationen zustande

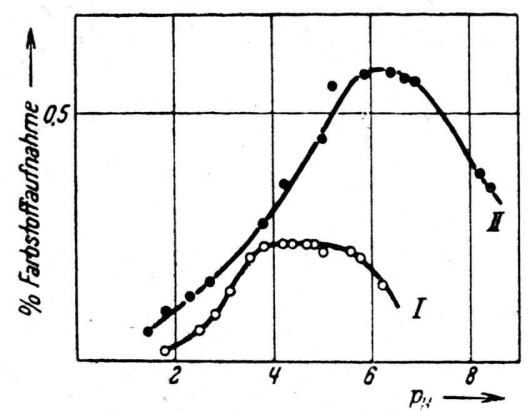

Abb. 6. Neutralrot. Farbstoffspeicherung in Gelatine (I) und Cellulose (II).

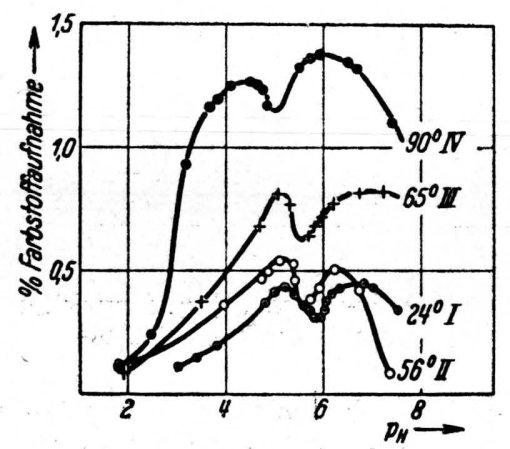

Abb. 7. Neutralrot. Farbstoffspeicherung in lebenden (I) und in durch Hitze geschädigten Hefezellen (II-IV).

kommt, liegt das $p_{k}$ bei diesem Wert $\left(p_{\mathrm{H}} 2,8\right)$. Die Base liegt erst oberhalb $p_{\mathrm{H}} 9,2$ ausschließlich vor. Mit Hilfe dieser Daten wurden die Dissoziationskurven für das Neutralrot konstruiert (s. Abb. 5 II u. III)*. Das von D rawe $\mathrm{rt}^{10}$ wiedergegebene Dissoziationsschema, welches von biologischer Seite allgemein als Diskussionsgrundlage für die Deutung des Speicherungsmechanismus herangezogen wird, kann nach diesen Untersuchungen als überholt angesehen werden.

Die Speicherungskurven für die beiden Modellsubstanzen Gelatine (I) und Cellulose (II) zei-

* Ähnliche Verhältnisse dürften übrigens auch bei dem früher von mir untersuchten Acridinorange vorliegen. Die Aufladung zum zweifach positiv geladenen - rot gefärbten - Farbstoffion vollzieht sich jedoch noch weiter im sauren Gebiet. gen das in Abb. 6 wiedergegebene, für einen basischen Farbstoff gewohnte Bild. Es sei besonders auf den großen Unterschied in der Höhe der Speicherungsfähigkeit dieser beiden Substanzen hingewiesen. Ebenso wird ersichtlich, daß etwa in dem Gebiet zwischen $p_{\mathrm{H}} 3,5-6$, in welchem die Kurve für die Gelatine gleichbleibend flach verläuft, die sterische Aufnahmefähigkeit für den Farbstoff offenbar erschöpft ist.

Der Verlauf der Speicherungskurven für lebende und tote Hefe (Kurve I und IV in Abb. 7) zeigte eine überraschende Unstetigkeit. Es ergab sich ein scharf ausgeprägtes Minimum, welches bei der lebenden Hefe bei $p_{\mathrm{H}} 5,9$ und bei der toten bei $p_{\mathrm{H}} 4,9$ lag. Diese Werte zeigen nun eine gute Übereinstimmung mit denjenigen für den IEP des Hefeprotoplasten (lebend: 6,2 und tot: 4,8), welche kaum eine rein zufällige sein dürfte. Um die Identität dieser „Lücke“ mit dem IEP nachzuweisen, wurden lebende Hefezellen auf einem siedenden Wasserbad unter dauerndem Rühren auf 56 bzw. $65^{\circ} \mathrm{C}$ erhitzt. Die Speicherungskurven der so geschädigten Hefesuspensionen zeigten eine Verschiebung der Lü̈cke auf $p_{\mathrm{H}} 5,7$ bzw. 5,5 (Kurve II u. III), welche der früher gefundenen Verschiebung des IEP ${ }^{1}$ gleichzusetzen ist. Die mit Acridinorange durchgeführte fluoreszenzmikroskopische Vitalitätskontrolle (vgl. Strugge ${ }^{11}$ ) zeigte, daß bereits die unbehandelte frische Hefe nekrobiotische und vereinzelt auch tote Zellen enthielt, welche beim Erhitzen auf $56^{\circ} \mathrm{C}$ in erhöhtem Maße auftraten, und mehr noch bei $65^{\circ} \mathrm{C}$. Die bereits bestehende Schädigung der frischen Hefe (in den heißen Sommermonaten war nur solche zu erhalten) erklärt so die veränderte Lage ihres IEP. In der auf $90^{\circ} \mathrm{C}$ erhitzten Hefesuspension waren nach Anfärbung mit Acridinorange nur noch rot fluorochromierte (=tote) Zellen enthalten.

Das Auftreten der Speicherungslücke mag seinen Grund darin haben, daß die Plasma-Eiweißkörper im IEP ihr Quellungsminimum besitzen und dadurch die rein räumlichen Bedingungen für den Farbstoff ungünstiger werden. Daß bei der Gelatine ein solches Minimum nicht nachzuweisen war, kann durch ihre weniger große Reaktionsbereitschaft begründet werden; die Feinstrukturen kommen deswegen nicht zum Ausdruck, oder sind wegen der Meßfehlergrößenordnung nicht erkennbar. Die Methode der quantita-

10 H. Drawe r t, Flora 34, 159 [1940].

11 S. Strugger, Flora 37, 73 [1943]. 
tiven Farbstoffspeicherungsmessung erweist sich somit als ein brauchbares Mittel zur weiteren Erforschung der physikalischen Chemie des Protoplasmas.

Nachdem so die Grundlagen zur Kenntnis des Farbstoffes und seiner Speicherung erarbeitet worden waren, konnte darangegangen werden, die Deutung der Speicherungsvorgänge und der damit zusammenhängenden Erscheinungen, die in der Literatur beschrieben worden sind, einer Revision zu unterziehen.

Strugger ${ }^{12}$ unternahm den Versuch, die auffällige Tatsache $\mathrm{zu}$ klären, daß - auf Grund einer Nichtfärbbarkeit des Cytoplasmas - vom Botaniker das Neutralrot als ausgesprochener Vakuolenfarbstoff angesehen wurde, während der Zoologe den gleichen Farbstoff zur Färbung des Cytoplasmas verwandte. Die Feststellung, daß auch im Cytoplasma pflanzlicher Zellen eine Speicherung des Farbstoffes erfolgt, ist Struggers Verdienst; da die Speicherung nur fluoreszenzoptisch nachweisbar ist, war sie bisher der Beobachtung entgangen. Die von Strugger durchgeführte Fluoreszenzanalyse vital gefärbter Epidermiszellen von Allium cepa ergab folgendes Bild:

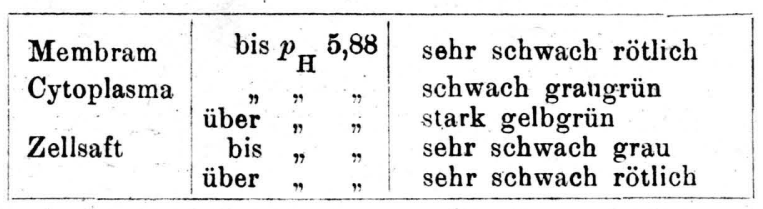

Auf Grund vergleichender Modelluntersuchungen macht Strugger die Farbkationen verantwortlich für die rötliche, und die in einem organischen Lösungsmittel gelöste Base für die stark gelbgrüne Fluoreszenzfarbe. Er kommt deswegen zu dem Schluß, daß in Membran und Zellsaft der Farbstoff in Form seiner Ionen, im Cytoplasma - und zwar dort in den Lipoiden - als Farbbasenmolekül gespeichert wird.

Es erscheint nun aber unwahrscheinlich, daß das Neutralrot als ein basischer Farbstoff nicht vom pflanzlichen Plasma elektrostatisch adsorbiert wird, nachdem tierisches in so hohem Maße dazu befähigt ist. Ferner ist es auffällig, daß, im Gegensatz zum lebenden, totes pflanzliches Plasma imstande ist, die Ionen des Farbstoffes zu speichern. In vollkommener Analogie zum Acridinorange nehme ich vielmehr an, daß es sich bei

12 S. Strugger, Protoplasma 34, 601 [1934]. dem unterschiedlichen färberischen Verhalten von lebendem und totem pflanzlichen Plasma gegenüber Neutralrot nicht um einen qualitativen, sondern lediglich um einen quantitativen Effekt handelt. Strugger selbst stellte fest, daß die Fluoreszenzfarbe wäßriger Neutralrotlösungen sich mit der Konzentration von dunkelrot-orange über rötlichgelb nach grau ändert. Er hat dieser Erscheinung keine große Bedeutung beigemessen, da die Fluoreszenzintensität in allen Fällen gering ist. Nun ist es aber durchaus denkbar, daß diese durch Adsorption wesentlich gesteigert werden kann, oder - wie es beispielsweise vom Auramin bekannt ist - die Fluoreszenz überhaupt erst ermöglicht. Diese Auffassung konnte durch folgende Versuche belegt werden.

In einer mit dest. Wasser angesetzten Neutralrotlösung $(c=0,25 \cdot 10-3 \mathrm{Mol} / l)$ wurde etwas pulverisierte Gelatine geschüttet, die sofort nach ihrer vollständigen Benetzung (etwa 20-30 Sek.) zur fluoreszenzoptischen Untersuchung gelangte. Die kleinen Gelatinepartikelchen zeigten in diesem Anfärbungsstadium an den Rändern eine schwach dunkelrote Fluoreszenzfarbe, die zur Mitte immer heller und gelber wurde, bis schließlich in der zentralen Region des Teilchens eine starke Grünfluoreszenz zu beobachten war. Wurde nach einigen Min. ein Tropfen der gleichen Suspension entnommen und untersucht, so fluoreszierten die Teilchen gleichmäßig schwach dunkelrot. Auch der umgekehrte Versuch gelang: Grobe Gelatinepartikelchen wurden in gleicher Weise wie oben angefärbt. Sie wurden daraufhin in eine Mikrokataphorese-Kammer gebracht und der Wirkung eines schwachen elektrischen Feldes unterworfen. Die gleichzeitige fluoreszenzmikroskopische Beohachtung ergab zunächst ein gleichmäßiges, schwach dunkelrotes Leuchten der Gelatine, die wegen der Größe ihrer Teilchen am Boden sedimentiert war. Nach einigen Min. war bereits ein deutliches Hellerwerden der Fluoreszenzfarbe, zunächst an den Rän= dern, dann auch in der Mitte der Teilchen, zu beobachten, die nach etwa $45 \mathrm{Min}$. in eine starke gelbrote bis gelbgrüne Farbe überging. Das elektrische Feld überwand also die Adsorptionsbindung des Farbstoffes mit der Gelatine, und es erfolgte wiederum die Trennung der beiden Bindungspartner, was die allmähliche Änderung der Fluoreszenzfarbe zur Folge hatte ${ }^{1}$.

Wir haben hier also eindeutig einen „Konzentrationseffekt" vor uns, und es ergibt sich die interessante Tatsache, daß das Neutralrot eine enge physikalisch-chemische Verwandtschaft mit dem Acridinorange aufweist. Diese Beobachtung dürftê den Anreiz bieten, weitere systematische Untersuchungen von biologischer wie auch physikalisch-chemischer Seite anzustellen. 
Wir sehen also, daß auch ein System, welches keine Lipoide enthält, das gleiche fluoreszenzmikroskopische Bild liefern kann wie das Cytoplasma. Somit werden wir zu der Tatsache geführt, daß auch das lebende Plasma imstande ist, Farbstoffionen des Neutralrots zu speichern. Neben einer mehr oder weniger großen Speicherung der Base in den Lipoiden, wie sie von Strugger ${ }^{13}$ für das Pyronin eindeutig bewiesen - werden konnte, haben wir also die schwach graugrüne Fluoreszenz des Cytoplasmas als eine sehr geringe Aufnahme von Farbstoffionen aufzufassen, während die stark gelbgrüne eine erhöhte Speicherung bedeutet, die aber noch nicht ausreicht, um eine im Hellfeld sichtbare Färbung hervorzurufen; diese tritt erst bei toten Zellen auf, welche die bereits früher beschriebenen tiefgreifenden plasmatischen Anderungen durchlaufen haben ${ }^{1}$. Die schwach dunkelrote Fluoreszenz der Membran und Vakuole schließlich zeigt eine starke Speicherung von Farbstoffionen an, welche die Konzentrationsauslöschung der Fluoreszenz zur Folge hat. - Besonders bemerkenswert bleibt die Tatsache, daß lebendes tierisches und pflanzliches Plasma den Farbstoff in so unterschiedlicher Höhe zu speichern vermögen, was auf strukturelle plasmatische Verschiedenheiten zurückgeführt werden muß.

Es sei an dieser Stelle noch auf eine weitere, sehr interessante Beobachtung der gleichen Arbeit Struggers eingegangen. Er plasmolysierte vakuolengefärbte $\mathrm{Zwiebel-Epidermiszellen} \mathrm{und} \mathrm{setzte}$ diese dem UV-Erregerlicht des Fluoreszenzmikroskopes aus. Die anfänglich schwach dunkelrote Fluoreszenz ging dabei im Laufe einiger Min. in ein helles Grüngelb über. Strugger schließt hieraus auf den Übergang von Farbstoffionen in Moleküle. Nachdem aber schon früher bereits Strugger ${ }^{14}$ durch Sauerstoffabschluß, und später Drawert ${ }^{15}$ durch elektrische Reizung von neutralrotgefärbten Zwiebel-Epidermiszellen die allmähliche Entfärbung der Vakuolen erreichen konnten, möchte ich die von Strugger beobachtete UV-Wirkung im gleichen Sinne erklären: Infolge einer durch extreme Außenbedingungen verursachten physiologischen "Umstimmung" des Zellsaftes (s. u.) wird der Farbstoff aus seiner Bindung in Freiheit gesetzt und wandert allmäh-

13 S. Strug ger, Flora 35, 101 [1941].

14 S. Strugger, Protoplasma 26, 56 [1936].

15 H. D r awer t, Planta 26, 391 [1937]. lich aus der Vakuole aus. Hiermit muß sich auch die konzentrationsabhängige Fluoreszenzfarbe ändern. Wir haben hier also den Gelatine-Modellversuch in vivo vor uns.

In einer neueren Arbeit $\mathrm{Höflers}{ }^{16}$ kommt die Verwandtschaft zwischen Neutralrot und Acridinorange auch im rein färberischen Verhalten klar zum Ausdruck. Diese Arbeit befaßt sich u. a. auch eingehend mit einer neuen Theorie der Vakuolenfärbung, die aber sicher nicht aufrecht erhalten werden kann. Höfler beobachtete, daß Alliumzellen, die, mit Acridinorange angefärbt, eine rote Fluoreszenz ihrer Zellsäfte aufweisen, sich in „leicht alkalischen“, ungefärbten Pufferlösungen nicht auswaschen lassen (vgl. dagegen Strugge $\mathrm{r}^{14}$ ), und nimmt das als Beweis dafür, daß Ionen nicht durch das Plasma exosmieren können. Bei der Einwirkung von $\mathrm{NH}_{3}$-Lösungen hingegen entfärbten sich die Vakuolen, was Höfler durch die Umwandlung der zunächst vorliegenden Farbstoffionen in Moleküle erklärt, die ihrerseits permeieren könnten. Höfler stellt also der Impermeabilität von Ionen die Permeabilität von Molekülen gegenüber.

Daß Ionen permeieren können, geht schon aus einem von Strugger $\mathrm{r}^{17}$ angestellten Versuch hervor. Er legte eine mit Acridinorange vakuomgefärbte Zelle in eine saure Pufferlösung, worauf die Entfärbung der Vakuole eintrat. Ein Versuch mit Neutralrot verlief in gleicher Weise (Strugger $\left.{ }^{18}\right)$. Diese Entfärbung läßt sich nur dadurch erklären, daß der in der Vakuole in Form seiner Ionen vorliegende Farbstoff permeieren kann. Es erscheint unmöglich, irgendwelche physikalischchemischen Bedingungen in der Zelle anzugeben, welche die Farbstoffionen in die undissoziierten Basenmoleküle überführen, die sich dann durch die Grenzfläche hindurchlösen könnten, um auf der anderen Seite wiederum als Ionen zu erscheinen. Höflers Versuch mußte anders ausfallen, da der $p_{\mathrm{H}}$-Wert seiner Auswaschlösung sich gegenuiber demjenigen der Farblösung nicht wesentlich geändert hatte und die Speicherungsbedingungen für den Farbstoff in der Vakuole infolgedessen ebenfalls nicht verändert wurden. Wird dagegen die Alkalität des Zellsaftes mit $\mathrm{NH}_{3}$-Lösungen so weit getrieben, daß die $C_{\mathrm{H}^{*}}$ sich in das Gebiet undissoziierter Farbbasenmoleküle verschiebt, so

${ }_{16} \mathrm{~K} . \mathrm{H}$ öfler, Mikroskopie 2, 13 [1947].

17 S. S tr u g g e r, Jen. Z. Naturwiss. 73, 97 [1940].

18 S. Stru.g ger, Protoplasma 24, 108 [1935]. 
wird die Bindung der Ionen rưtckgängig gemacht. Die nunmehr gebildeten freien Moleküle finden naturgemäß ein starkes Konzentrationsgefälle vor, welches von ihnen durch Permeation ausgeglichen wird. Außerdem gibt es Zellen, die bereits im stark sauren Milieu eine Anfärbung ihrer Vakuolen mit Acridinorange und Neutralrot erfahren (s. u.), und zwar in einem $p_{\mathrm{H}}$-Gebiet, in welchem ausschließlich Farbstoffionen zur Verfügung stehen, die also nur als solche in den Zellsaftraum gelangen können. Schon nach diesen Uberlegungen scheint es ungerechtfertigt, die Impermeabilität von Ionen allgemein zu postulieren.

Ohne weiter auf Einzelheiten der Höflerschen Theorie einzugehen, sei dagegen folgendes festgestellt: Die Speicherung eines primär oder sekundär - bedingt durch die Acidität des Zellsaftes dissoziierten, basischen Farbstoffes in der Vakuole kann zustandekommen: 1. durch eine chemische Bindung mit Gerbstoffen oder dergl., 2. durch Lösung in Fettsäuren und 3. durch elektroadsorptive Bindung an Kolloide im Sinne einer vollkommenen Parallele zum Speicherungsvorgang im Plasma. Diese letztere Möglichkeit ist bisher außer acht gelassen worden. Da der Zellsaft in mehr oder weniger hohem Maße Kolloide enthält (vgl. Frey-Wyssling ${ }^{19}$ ), ist die theoretische Möglichkeit einer elektrostatischen Adsorptionsbindung mit Farbstoffen gegeben. Wir müssen in Verfolgung dieser Theorie also bei bestimmten Zellen auch von einem IEP des Zellsaftes bzw. einem $\operatorname{IEP}_{\mathbf{M}}$ (Mittelwert aller IEP) seiner umladbaren kolloiden Bestandteile sprechen und kommen zu dem Schluß, daß eine Farbstoffspeicherung in diesem Sinne unter Voraussetzung entsprechender elektrischer Bedingungen möglich ist. Für gewisse Zelltypen ergibt sich die Erklärung dafür, daß der $p_{\mathrm{H}^{-}}$Wert des Beginns der Vakuolenfärbung mit Acridinorange und Neutralrot beim gleichen Objekt gleich ist. Hiermit wird die Struggersche Ansicht ${ }^{14}$ bestätigt, „,daß für die Lage des Umschlagspunktes" von der Membran- zur Vakuolenfärbung ,nicht etwa eine Konstante des Farbstoffes, sondern bestimmte Eigenschaften der Zelle verantwortlich sind".

Höfler unterscheidet zwischen ,vollen“ und „leeren“ Zellsäften; in vollen tritt nach seiner

10 A. Frey-Wy ssling, Submikroskopische Morphologie des Protoplasmas und seiner Derivate, Bornträger, Berlin 1938, S. 194.
Theorie Speicherung durch chemische Bindung an zelleigene Stoffe ein, in leeren dagegen ist die Speicherungsursache die Funktion der Vakuole als "Ionenfalle“. Der erstgenannte Typus zeigt, mit Acridinorange angefärbt, eine grüne bis gelbgrüne Fluoreszenzfarbe, der zweite eine rote. Daß die gelbgrüne Fluoreszenz derjenigen des chemisch gebundenen Farbstoffes zukommt, ist eine unbewiesene Annahme. Das Auftreten dieses Farbtones läßt sich weniger zwangvoll mit dem ,Konzentrationseffekt" in Zusammenhang bringen. Da sich leere Zellsäfte ohne weiteres als speicherungsfähiger erweisen können als volle, so können diese Begriffe leicht Anlaß zur Verwirrung geben. Ich sehe aber ohnehin große Schwierigkeiten bei der Aufrechterhaltung der Höflerschen Theorie und möchte deswegen bei der Einteilung der Zelltypen nach ihren Vakuolen-Inhaltsstoffen bereits geläufige Begriffe beibehalten. Danach können Zellsäfte unterschieden werden als vorwiegend: 1. gerbstofführend, 2. fettsäurereich und 3. wäßrig und kolloid. Die Speicherungsvorgänge zu 1. und 2. sind ohne weiteres verständlich. $\mathrm{Zu}$ 3. möchte ich nun der obigen Vorstellung gemäß folgendes ausführen: Wäßrig sind Zellsäfte, die - bei Fehlen von Gerbstoffen und Fettsäuren - wenig farbstoffbindungsfähige Kolloide enthalten. Theoretisch läßt sich voraussagen, daß solche Zellsäfte keine Speicherung im eigentlichen Sinn aufweisen können. Da keine oder nur geringe Adsorptionsmöglichkeiten bestehen, kann sich deswegen auch in einer solchen, mit Acridinorange gefärbten Vakuole kein Konzentrationseffekt ausbilden, ebenso wie die ,elektrische Schwelle" nicht vorhanden ist. Diese Zellsäfte vermögen deswegen auch den Farbstoff aus stark saurem Milieu aufzunehmen. Höfler fand in Platanthera bifolia einen solchen Zelltypus, der im gesamten sauren bis alkalischen Gebiet, mit Acridinorange angefärbt, eine grüne Fluoreszenz der Vakuole zeigte; hier handelt es sich in überwiegendem Maße um einen weitgehend vom $p_{\mathrm{H}^{-}}$ Wert unabhängigen Lösungsvorgang des Farbstoffes. Die kolloiden Zellsäfte dagegen speichern die Farbstoffkationen elektro-adsorptiv oberhalb ihres IEP und können infolge Eintretens des Konzentrationseffektes rot fluorochromiert sein. Die hierbei auftretende Vakuolenkontraktion verstärkt darüber hinaus noch diesen Effekt. Eine Erklärung für die Kontraktion selber liegt insofern nahe, als durch die Adsorption der Farbstoff- 
ionen die elektrischen Verhältnisse der Zellsaftbestandteile weitgehend verändert (neutralisiert) werden. Diese Erscheinung läßt sich also mit der oben gemachten Feststellung in Parallele setzen, wonach die Quellung der Plasma-Eiweißkörper im IEP ein Minimum besitzen. Die Beobachtung, daß die Entfärbung der Vakuole wiederum mit einer Aufhebung der Kontraktion verknüpft ist, zeigt den ursächlichen Zusammenhang zwischen Speicherung und Kontraktion (vgl. Strugger ${ }^{14}$ u. a.). Die Kontraktion wird hiernach nicht durch ein Zusammendrücken, etwa durch Quellung des Plasmas, sondern durch ein aktives Zusammenziehen (Entquellung) der Vakuole verursacht. Diese Erklärung kann vorerst nur für die reversible Vakuolenkontraktion gelten, die bei der Vitalfärbung beobachtet wird; der durch Wundreiz $u$. ä. verursachten Kontraktion liegt eine bisher noch unbekannte Mechanik zugrunde.

Zwischen dem Typus des kolloiden und dem des wäßrigen Zellsaftes gibt es naturgemäß alle Übergänge. Es liegt auf der Hand, daß auch ein und dieselbe Zelle im Verlauf verschiedener physiologischer Stadien (Alter) von einem zum anderen Typus wechseln kann. Außerdem komplizieren sich die Verhältnisse noch mehr dadurch, daß der IEP der Vakuolen Schwankungen unterworfen ist, die durch den Stoffwechsel der Zelle unter besonderen Bedingungen hervorgerufen, werden können. Zum Beleg der ersten Möglichkeit sei auf die. Untersuchungen Strug gers ${ }^{20}$ an jungen Keimwurzeln von Triticum vulgare hingewiesen, wo sich das Eintreten des Farbstoffes in die Vakuole $p_{\mathrm{H}^{-}}$abhängig, je nach dem Alter der Zellen, vollzog, während als Beispiel für die zweite angeführte Möglichkeit der Umstimmung des Zellsaftes eine andere Beobachtung $\mathrm{Strug} g \mathrm{ers}^{14}$ angegeben sei, wonach vakuomgefärbte Epidermishäutchen von Allium bei Sauerstoffabschluß nach kurzer Zeit ihren Farbstoff an die Membranen abgeben und sich dadurch entfärben. Auch die gleiche Beobachtung von $\mathrm{Draw}$ - $\mathrm{t}^{15}$ an dem gleichen Material nach elektrischer Reizung sei hier eingereiht, ebenso wie die von Strugger ${ }^{12}$ beobachtete Anderung der Fluoreszenzfarbe nach Bestrahlung mit UVLicht. Wir können also nunmehr auf Grund des färberischen Verhaltens der Vakuole bestimmte Angaben über ihren Inhalt machen und gegebenen-

20 S. Strugger, Arch. exp. Zellforschg. 19, 199 [1937]. falls ebenso Rückschlusse auf komplizierte Stoffwechselvorgänge ziehen.

Es sei ausdrücklich betont, daß diesen hier in großen Zügen entwickelten theoretischen Vorstellungen keinerlei eigene Experimente zugrunde liegen. Doch sind Versuche zu diesem speziellen Problem so zahlreich in der Literatur beschrieben, daß schon ihre Sichtung zu dieser Deutung ihrer Vorgänge geführt hat. Die in dieser Arbeit yorliegenden quantitativen Untersuchungen, und vor allem auch die früher gewonnenen Meßergebnisse über die Speicherung des Acridinorange, ließen aber die große Bedeutung der elektrischen Verhältnisse der Zelle für die Speicherung erkennen und führten schließlich dazu, die Erklärung für die Ursachen der Vakuolenfärbung und der damit zusammenhängenden Erscheinungen in dieser Richtung zu suchen. Die neuerdings von $\mathrm{H}$ öfle $\mathrm{r}^{16}$ aufgestellte Theorie befriedigt jedenfalls nicht; es bleibt abzuwarten, ob die Richtigkeit der hier gegebenen Deutungen durch weitere exakte Untersuchungen bestätigt werden kann.

\section{K a t a p horese}

Bei den früher angestellten mikrokataphoretischen Untersuchungen an Hefezellen konnte festgestellt werden, daß die anodische Wanderung der ungefärbten Zellen durch die negative Ladung der Zellmembran verursacht wurde ${ }^{1}$. Diese Ladung konnte durch Acridinorange-Kationen abgesättigt werden, so daß nunmehr die der $C_{\mathrm{H}}$ des Außenmediums entsprechende freie Ladung des Plasmas die Wanderung der Zellen bestimmte. Hiermit war eine Methode gefunden, die eine Bestimmung des IEP des Protoplasten ermöglichte. Um den Einfluß von Farbstoffionen auf Hefezellen für die Wanderung im elektrischen Feld weiterhin zu studieren, wurden gleiche Versuche mit den in dieser Arbeit untersuchten drei Farbstoffen angestellt.

Rhodamin B. Bei den mit Rhodamin B angefärbten Hefezellen war zu erwarten, daß der oben angegebene Effekt nicht so auffällig in Erscheinung treten konnte, da beim Rhodamin nur im stark sauren Gebiet Farbstoffkationen zur Verfügung stehen. Nur in diesem Gebiet wäre also eine Neutralisation der elektrischen Ladung der Membran durch die Farbstoffionen möglich, doch konnte auch hier keine - der Ladung des Plasmas gemäße - kathodische Wanderung beobachtet werden. 
Eosin. Die Anfärbung von Hefezellen mit Eosin ergab keine Beeinflussung der bei allen $p_{\mathrm{H}^{-}}$Werten gleichen anodischen Wanderungsrichtung, was ohne weiteres unter Hinweis auf die saure Natur des Farbstoffes verständlich ist.

Neutralrot. Das Neutralrot zeigte auch bei der kataphoretischen Untersuchung gefärbter lebender und toter Hefezellen eine völlige Parallele zum Acridinorange. Entgegen den bei oben angeführten Speicherungsmessungen verwendeten Farbstoffkonzentrationen wurden für diese Versuche die Farbstofflösungen mit der gleichen Konzentration wie bei den Versuchen mit Acridinorange $\left(c=0,5 \cdot 10^{-3} \mathrm{Mol} / l\right)$ angesetzt. Dieser Hinweis ist deswegen wichtig, da eine Abhängigkeit der Farbstoffspeicherung in der Zellwand von der Konzentration der Farblösung in gewissen Grenzen möglich erscheint; doch soll die quantitative Seite dieser Frage erst später bearbeitet werden. Deŕ IEP der hitzegetöteten Hefe ergab nun denselben Wert $\left(p_{\mathrm{H}} 4,9\right)$, wie er früher bei
Anfärbung mit Acridinorange erhalten wurde. Für die frische Hefe ergab sich ein IEP von 5,6. Dieser Wert liegt erheblich unter demjenigen, der mit Hilfe des Acridinorange gefunden wurde $\left(p_{\mathrm{H}} 6,2\right)$. Wie oben bereits ausgeführt, hat das wohl aber seinen Grund in der schon bestehenden Schädigung der frischen Hefe, die auch fluoreszenzoptisch nachgewiesen werden konnte. Interessant ist dabei das gute Utbereinstimmen dieses Wertes mit der „Lücke“ (s. Abb. 7).

Durch diese Untersuchungen wird die Möglich: keit bestätigt, mit Hilfe einer durch Anfärbung mit geeigneten Farbstoffen erreichten elektrischen Absättigung der Membran, den IEP des Protoplasten zu bestimmen. Diese Feststellung dürfte von einigem methodischen Wert sein. Es ist geplant, in einer späteren Arbeit ausführlich die Kataphorese von Mikroorganismen zu behandeln.

Hrn. Prof. Dr. G. Scheibe, München, danke ich freundlichst für die wertvolle Unterstützung bei der theoretischen Klärung der Dissoziationsverhältnisse der untersuchten Farbstoffe.

\title{
Untersuchungen zum Wesen der Erholungsatmung
}

\author{
Von Otto HaRnisch \\ Aus der Hydrobiologischen Anstalt der Max-Planck-Gesellschaft zu Plön \\ (Z. Naturforschg. 3 b 453-457 [1948]; eingegangen am 8. Oktober 1948)
}

\begin{abstract}
Um die Frage zu entscheiden, ob die Abhängigkeit der gesteigerten Erholungsatmung vom $\mathrm{O}_{2}$-Partialdruck des Mediums rein physikalisch dadurch zu erklären ist, daß infolge des erhöhten $\mathrm{O}_{2}$-Verbrauchs der ,kritische Punkt“ der Druck-Verbrauchskurve in höhere Partialdruckbereiche verlagert ist, oder ob die Erholungsatmung eine besondere, prinzipiell vom $\mathrm{O}_{2}$-Partialdruck abhängige Komponente enthält, wurde der Einfluß stark erhöhten $\mathrm{O}_{2}$-Partialdrucks auf durch erniedrigte Temperatur erheblich herabgesetzte Erholungsatmung untersucht. Es ergab sich durchweg, daß die Verminderung der Größe der Erholungsatmung den Einfluß der Erhöhung des $\mathrm{O}_{2}$-Partialdrucks auf die Atmungsgröße nicht zum Verschwinden bringt oder vermindert, sondern ihn sogar verstärkt. Hieraus ist zu folgern, daß die Erholungsatmung eine besondere Komponente (die sekundäre Oxybiose) enthält, die prinzipiell vom $\mathrm{O}_{2}$-Partialdruck des Mediums abhängig ist, und die möglichst rasche Erledigung eines durch vorangegangene anaërobe Prozesse notwendig gewordenen oxybiotischen Prozesses leistet.
\end{abstract}

I n einer Reihe von Arbeiten ${ }^{1}$ habe ich den Stand1 punkt vertreten, daß in der ,Erholungsatmung“", die im Anschluß an Prozesse anaërober Energiegewinnung abläuft, ein Prozeß wesentlich anderen Charakters vorhanden sei als in der noimalen Oxybiose der Standardenergiegewinnung;

1 O. H a r n i s ch, Z. vergl. Physiol. 22 [1935]; 24 [1937]; Verh. dtsch. zool. Ges. 1935, 1937; Naturwiss. 29 [1941]; Arch. Hydrobiol. 60 [1943].
Sein wesentlichstes Merkmal erblickte ich in der prinzipiellen Abhängigkeit seiner Größenordnung vom $\mathrm{O}_{2}$-Partialdruck des Mediums, also in der Ungültigkeit des Alles- oder Nichts-Gesetzes der Atmung. Hiergegen hat v. Budde $\mathrm{nbrock}^{2}$ ein sehr wohl begründetes Bedenken geäußert: er sieht in der von mir beobachteten Abhängigkeit

2. . B udden bock, Grundriß d. vergl. Physiol., Berlin 1939. 